\title{
Activities through which Young Spend Leisure Time in Hamadan: Effects of Personal Values and Attitudes
}

\author{
Sajjad Sedighi \\ PhD student in Assessment \& Measurement, Allameh Tabataba'i University, Iran, Tehran \\ Email: direct2.2006@gmail.com \\ Mohammad Daraie
}

M.A. in History and Philosophy of Education,Payame Noor University, Iran, Hamedan Email: Daraie611@yahoo.com

Majid Babazadeh

M.A. in Educational Technology, Allameh Tabataba'i University, Iran, Tehran Email: majidbabazadeh@yahoo.com

Hassan Qarabaghi

Ph.D in Educational Technology and a member of the faculty of Malayer University, Iran, Malayer Email: H.qarabaghi@malayeru.ac.ir

\section{Doi:10.5901/mjss.2016.v7n1s1p74}

\section{Abstract}

The present study aimed to examine how young people in Hamadan City spend their leisure time with an emphasis on their personal values and attitudes and to investigate its relationship with watching TV, watching satellite channels, using computer, using the Internet, using library and doing sports. The research population consisted of the young population in Hamadan Province. The sample size was computed using Cochran's formula. The results showed that over 60 percent of the participants considered personal values and attitudes as an important factor in how people spend their leisure time. However, there was only 14 percent congruity between the existing leisure activities and personal values. The results of ANOVA revealed the strongest correlation of personal values and attitudes with doing leisure time sports.

Keywords: attitude; leisure time; pathology; personal values; young people

\section{Introduction}

The modern world is facing new challenges and complicated issues the majority of which originates from industrial developments and increasing complexities. Leisure time practice is one of these issues so that leisure time practices exert a marked impact on personal and social life of young people and adolescents. Many humanities scholars have focused on diverse functions of leisure time (Vasefian et al., 2014). On the other hand, leisure time is an opportunity to do free activities which people do voluntarily with interest and enthusiasm. People with different talents seek their suitable leisure activities and work toward it. Thus, leisure time may help nurture an individual's personality and empower him as a creative and active person to do his part (Moshfeghi, 2012).

Youth is the prime time of human life and the best opportunity for contribution to individual and social domains. Young people are considered as the most invaluable resources and assets of a society. The development of any nation depends on a young generation enjoying health, joviality, creativity and innovation as a result of education and empowerment (Author, 2013). Leisure time is a scientific research area that is perceptible only via comprehensive research. Some scholars regret that leisure time is addressed only through the lens of educational sociology. Accordingly, they believe that basic, moral and religious discussions are missing in leisure time research (Tipa, 2009). In the 1970s and 1980s, the sociologists in the western countries promoted research on leisure time mainly because politicians showed interest in the issue. In their studies, they voiced concern that people may indulge themselves in leisure time in 
near future (Pur Soltani, 2010).

In the past, leisure time practice was not an issue of interest as a social phenomenon due to traditional life, low technology, deep emotional attachment among people and extended families. However, marked changes have occurred in the structure and texture of families due to increasing industrial development, mass media and cultural changes so that extended families have transformed into nuclear families. In the current communication age in which moments and seconds compete against each other and media have emerged, people have fewer opportunities for direct cooperation and communication than in the past. New jobs have resulted in more individualism. In effect, social and cultural changes have directed the attention of authorities and experts toward leisure time (Author, 2014). Leisure time constitutes an important part of daily life. Considering the increasing diversity of urban leisure activities, people make choices as to how to spend their leisure time. This selection is not made freely though. It is subject to a number of factors such as age, gender, social class and personal tastes, which are the key factors affecting the selection of a given leisure activity (Hesam, 2008). Research on leisure time has shown that although leisure activities are distinct from social, family, peer and the ruling system expectations, they may not be done without them. In other words, various factors determine how to spend one's leisure time (Rajab Zadeh, 2002). Besides, different lifestyles hinge on the way leisure time is spent. Therefore, economic status, socio-cultural attitudes, time, space, age, gender, social class and personal tastes and interests contribute to how people may spend their leisure time (Azani \& Ahmad Zadeh, 2012).

Literally, leisure refers to comfort and convenience as opposed to daily struggles and preoccupation that cause fatigue (Kohestani, 2009). In 'Leisure in America: a social inquiry', Max Kaplan (1960) indicates that leisure includes relatively-spontaneous activities and experiences that an individual has in his free time, which is psychologically reminiscent of good memories, potentially involves a broad range of participations including personality norms and requirements, and provides opportunities for recreation, personal development and helping people (p. 28). According to the International Sociological Association (ISA), leisure involves a set of activities an individual does voluntarily in order to repose, rejoice and broaden his knowledge or to receive non-profit education and have social participation after meeting occupational, family and social requirements (Beenackers et al., 2012). The sociology of leisure time has mainly focused on the advantages and disadvantages of leisure time definitions. By advantages and disadvantages, we do not mean the initial or superficial value-based stance. The disadvantage refers to time and considers the off-duty time as the leisure time. The advantage refers to the content and function of leisure time (Author, 2014).

Values mark a conviction about actions, conditions and behaviors that are considered superior to opposing actions, conditions and behaviors personally or socially. Value refers to whatever that is suitable, desirable, invaluable or appreciable for the individual or group. Indeed, value is the most fundamental factor in determining thought and action and shaping the social life of an individual. Thus, values are benchmarks that give meaning to culture and society (Farahani, 2010). Attitudes are a set of beliefs, emotions and behavioral intentions toward an object, person or event. In other words, they are a complex cognitive process used to explain human behavior and indicate a relatively-consistent interest in a person, thing or event demonstrated in feelings and behavior (Varzeshkar, 2009).

Leisure activity has values per se and does not follow an external purpose. Such activity is undertaken based on spontaneous and internal motivations but is not reliant on external factors. Researchers contend that leisure activities should inherently satisfy people so that participation in leisure activities should be induced by an internal motivation. Research has shown that internal motivation is directly associated with perceived competence (Wegner et al., 2008). IsoAhola (1999) observed that people often tend to do activities that bring about internal rewards; the most important of these rewards is perceived competence (p. 39). External motivation often decreases perceived freedom in people and exerts a negative impact on their participation in leisure activities. The commitment and satisfaction inherent in the internal motivation is much stronger than the participation induced by external factors (Christel, 2003). The positive impacts of a given leisure activity are demonstrated when the individual feels he can control or influence his leisure time. In fact, such feeling could be defined as a feeling of selection or self-determination. In other words, people not only need to be actively involved in planning or organizing an activity but also should feel they are in control or share the activity (Amir Fakhrian \& Moini, 2014).

\subsection{Research questions}

The study was conducted to address the questions: 'what is the impact of personal values and attitudes on leisure time practices?' and 'what is the relationship of personal values and attitudes with other leisure variables?'

To answer the above questions, the study adopted a descriptive approach and a survey design. The research population consisted of all young people in Hamadan City. 


\section{Method}

\subsection{Participants}

According to the latest report of Statistical Centre of Iran, the youth population in Hamadan Province amounts to 627442 people. The research sample was computed using Cochran's formula to be 141 people consistent with the proportion of Hamadan City population to the Hamadan Province population. Considering the sampling norms using Cochran's formula, the desirable level of significance was $95 \%$ consistent with previous studies.

\subsection{Materials and apparatus}

A researcher-made questionnaire was used to obtain the data. The questionnaire was developed based on the research objectives and consisted of closed items on a 5-point Likert scale. In addition to personal information, the questionnaire collected information on how people used TV programs, satellite broadcasts, computer, the Internet, library, sports facilities, environmental advertisements and organizational programs as well as on their lifestyle, religious beliefs, gender attitudes and family status. The content validity of the questionnaire was approved of by experts. The reliability of the scale was measured using Cronbach's alpha formula, which yielded a reliability coefficient of 0.73 .

\subsection{Data analysis}

Descriptive statistics including frequency and percentage were used to describe the data. Inferential statistics including $t$ test and ANOVA were run to analyze the data.

\section{Results}

Table 1 summarizes the demographic information of the participants.

Table 1. Participants' demographic information

\begin{tabular}{llc}
\hline Variable & & Value \\
\hline \multirow{2}{*}{ Gender } & Male & 32.4 \\
& Female & 67.6 \\
\hline \multirow{2}{*}{ Age group } & Maximum: 19-21 years (\%) & 66.7 \\
& Minimum: 25-29 years (\%) & 6.9 \\
\hline \multirow{2}{*}{ Marital status } & Single & 89.2 \\
& Married & 10.8 \\
\hline \multirow{3}{*}{ Education } & Diploma & 38.6 \\
& Bachelor's & 29.7 \\
& Master's & 3 \\
& Seminary & 22.8 \\
\hline
\end{tabular}

The results of descriptive statistics as to the impact of personal values and attitudes on leisure time practices showed that over $60 \%$ of the participants believed that their personal values and attitudes influenced their leisure time practices. This indicates the importance of personal values and attitudes in leisure time planning (Table 2).

Table 2. Impact of personal values and attitudes on leisure time practices

\begin{tabular}{lcc}
\hline Item & Frequency & percentage \\
\hline Very small & 4 & 3.1 \\
Small & 4 & 3.1 \\
Moderate & 35 & 28.9 \\
Large & 49 & 41.2 \\
Very large & 28 & 23.7 \\
\hline Total & 120 & 100 \\
\hline & & \\
& 76 &
\end{tabular}


Interestingly, only did $14 \%$ of the participants report that the existing leisure time facilities and activities corresponded to their personal values and attitudes while $48 \%$ of the participants reported a moderate consistency. Still, $37.8 \%$ of the participants believed that there was no consistency whatsoever between their personal values and attitudes and the existing leisure time facilities and activities in the society.

The results of ANOVA to test the relationship of personal values and attitudes with watching TV programs showed no significant correlation between differences in personal values and attitudes and watching TV programs ( $F(4$, 345)=1.556, $\mathrm{P}=0.186$ ). Thus, differences in personal values and attitudes do not contribute to the amount of watching TV programs. The results also revealed no significant correlation between differences in personal values and attitudes and considering state TV channels as suitable leisure time fillers $(F(4,341)=1.009, P=0.403)$.

The results of ANOVA also showed no significant correlation of differences in personal values and attitudes with watching foreign TV channels (satellite channels) to fill leisure time $(F(4,339)=0.269, P=0.898)$. Moreover, the findings demonstrated no significant correlation between differences in personal values and attitudes and considering foreign TV channels (satellite channels) as suitable leisure time fillers $(F(4,283)=0.579, P=0.678)$.

The results of ANOVA disclosed no significant correlation of differences in personal values and attitudes with using computer and computer applications to fill leisure time $(F(4,342)=0.460, P=0.765)$. The same was found about considering computer as a suitable leisure time filler. Accordingly, the results of ANOVA showed no significant correlation between differences in personal values and attitudes and considering computer and its applications as suitable leisure time fillers $(F(4,329)=2.267, P=0.062)$.

The results of ANOVA demonstrated no significant correlation of differences in personal values and attitudes with using the Internet to fill leisure time $(F(4,342)=0.254, P=0.907)$. The findings also showed no significant correlation between differences in personal values and attitudes and considering the Internet as a suitable leisure time filler $(F(4$, $322)=0.559, P=0.699)$.

The results of ANOVA showed no significant correlation of differences in personal values and attitudes with using library to fill leisure time $(F(4,344)=1.383, P=0.239)$. The findings also indicated no significant correlation between differences in personal values and attitudes and considering the library facilities as suitable leisure time fillers $(F(4$, $320)=2.3, P=0.059$ ).

The results of ANOVA showed a significant correlation of differences in personal values and attitudes with doing sports to fill leisure time $(\mathrm{F}(4,345)=2.446, \mathrm{P}=0.046)$. The findings also disclosed a significant correlation between differences in personal values and attitudes and considering sports as suitable leisure time fillers $(F(4,337)=1.409$, $\mathrm{P}=0.042)$.

\section{Discussion}

Leisure time is one of the phenomena addressed by many experts including sociologists, social science scholars and even ordinary people. Scholars contend that the cultural and social aspects of leisure time are of utmost importance. In fact, due to the all-encompassing nature of leisure time among different social groups, it can be studied from different aspects including from the viewpoint of personal values and attitudes. This study aimed to investigate how young people in Hamadan City spent their leisure time with an emphasis on personal values and attitudes.

The results showed that over $60 \%$ of the respondents believed that personal values and attitudes was a key factor contributing to leisure time practices. However, there was only $14 \%$ consistency between the existing leisure activities and personal values and attitudes. The results of $t$ test and ANOVA revealed a significant correlation between differences in personal values and attitudes and doing sports to fill leisure time. However, the same significance was not observed about other research variables. It seems that a lack of correlation of personal values and attitudes with watching state and satellite TV channels, using computer applications, the Internet and library results from their prevalence, commonness and availability.

\section{References}

Amir Fakhrian, M.,\& Moini, A. (2014). Tourism planning and leisure time practices in Mashhad with an emphasis on identifying leisure time practices among citizens. Journal of Applied Research in Geography, 14 (33).

Azani, M.,\& Ahmad Zadeh, M. (2012). Locating leisure time resorts using GIS: A case study in Marvdasht City. Geography Regional Planning Quarterly, 2.

Beenackers, M.A., Kamphuis, C., Giskes, K., Brug, J., Kunst, A.E., Burdorf, A.,\& van Lenthe, F.J. (2012). Socioeconomic inequalities in occupational, leisure-time, and transport related physical activity among European adults: A systematic review. Int J Behav Nutr 
Phys Act, 9:116-139.

Christel, A. (2003). How Women and Men spend Their Time. Statistics in Focus Themes.

Author (2013).

Author (2014).

Hesam, F. (2008). Revisiting the concept of citizenship and how to study citizenship in our cities. Tehran: Second Festival of Selecting the Best Researchers and Innovators in Urban Management.

Iso-Ahola, S.E. (1986). Psychology of Sports: A Social Psychological Approach. R. Fallahi, M. Hajilo (Trans.). Tehran: Organization for Physical Education.

Jalali Farahani, M. (2010). Management of leisure time and recreational sports. Tehran: Tehran University.

Kaplan, M. (1960). Leisure in America: a social inquiry. New York: Jon Wiley.

Kohestani, H.A. (2009). Research on leisure time and how to use leisure time efficiently. Mashhad: Tieh.

Moshfeghi, S. (2012). Leisure time in mentally-retarded children. Islamic Education Quarterly, 113: 52-56.

Pur Soltani Zarandi, H. (2010). Leisure time. Tehran: Bamdad.

Rajab Zadeh, A. (2002). Iranians' cultural behaviors: activity and consumption of cultural goods in Tehran. Tehran: Ministry of Culture and Islamic Guidance.

Tipa, M. (2009). Investigating the relationship between socio-economic status and leisure time practices among 18-29 year-old students at the college of social sciences, Allameh Tabatabaie University. Unpublished MS thesis, College of Social Sciences, Allameh Tabatabaie University, Tehran, Iran.

Varzeshkar, A. (2009). The comprehensive whole in managerial approaches. Tehran: Center for Management Studies.

Vasefian, F., Bagheri, M., Azadi Pur, Sh.,\& Vasefian, F. (2014). Investigating leisure time practices among students at Ardestan Islamic Azad University. Youth Sociological Studies Quarterly, 13: 145-156.

Wegner, L., Flisher, A., Chikobvu, P., Lombard, C., \& King, G. (2008). Leisure boredom and high school dropout in Cape Town, South Africa. Journal of Adolescence, 31(3): 421-31. 